\title{
Numerical Simulation of Falling Liquid Film Flow on a Vertical Plane by Two-Phase Lattice Boltzmann Method
}

\author{
Andreas Hantsch and Ulrich Gross \\ Institute of Thermal Engineering, TU Bergakademie Freiberg, G.-Zeuner-Str. 7, 09599 Freiberg, Germany \\ Correspondence should be addressed to Andreas Hantsch; hantscha@iwtt.tu-freiberg.de
}

Received 7 September 2012; Revised 4 December 2012; Accepted 18 December 2012

Academic Editor: Oronzio Manca

Copyright (C) 2013 A. Hantsch and U. Gross. This is an open access article distributed under the Creative Commons Attribution License, which permits unrestricted use, distribution, and reproduction in any medium, provided the original work is properly cited.

Falling liquid film flow is widely used in many processes. Supplementary to experimental studies, Navier-Stokes-based models have been employed for describing film flow phenomena. These models are often disadvantageous since they are either strongly limited in their generality or need enormous computational resources. In this investigation, a new approach is proposed for modelling flow by lattice Boltzmann methods. Therefore, the well-known Shan-Chen model (Shan and Chen, 1993) has been employed to an isothermal falling liquid film. The validity of the implementation has been checked against some single-phase and two-phase reference cases. Test series have been conducted for three different Reynolds numbers without external disturbances and for one Reynolds number with sinusoidally pulsating inlet velocity. The computational results show that lattice Boltzmann methods are capable to model falling liquid film flow and that the flow morphology is in qualitatively good agreement with other numerical and experimental works.

\section{Introduction}

Falling liquid film flow is a critical operation in many engineering processes, such as densification of suspensions or emulsions or the separation of mixtures, liquefaction in the condenser of a power plant or heat and mass transfer in a heat pipe. Experiments and previous simulations have shown that the film becomes wavy from certain Reynolds numbers $\operatorname{Re}=(u h) / v$ on (see [1-5]) and that waviness generally leads to a heat transfer augmentation.

The theoretical and numerical description of falling liquid films is still a challenge. Existing models are either limited in their generality or need enormous computational resources. These are evolution equations of the film thickness (also known as Benney equation, long-wave equation), (weighted residual) integrated boundary layer equations (IBL, WIBL, WRIBL), and the full Navier-Stokes equations, with increasing computational demand.

Based on the two-dimensional Navier-Stokes equations, evolution equations of the film thickness have been designed, by introducing a stream function and assuming long disturbance wavelengths and Reynolds number of the order of one.
First research has been performed by Yih, 1955 [6]; Yih, 1963 [7], Benjamin, 1957 [8], and Benney, 1966 [9], who derived the evolution equation for a film flowing down an inclined plane and investigated the stability of the film for certain conditions. In the last decades, additional physical effects have been implemented, such as phase change, thermocapillarity, non-Newtonian fluids, electromagnetic interactions, influence of ultrasound, and fully temperature-dependent properties (for an overview, see [10]).

Shkadov, 1967 [11] was the first to develop the integrated boundary layer equations from Navier-Stokes equations and qualitatively good agreement with experiments in the nonlinear regime was obtained. It fails, however, to represent the critical behaviour. Ruyer-Quil and Manneville, 1998 [12]; Ruyer-Quil and Manneville 2000 [13]; Ruyer-Quil and Manneville, 2002 [14] extended the model of Shkadov and created a new one, based on weighted residual integrated boundary layer equations (WRIBL), which represent experimental data much better. Nguyen and Balakotaiah, 2000 [15] gave a literature review and designed a model consisting of three partial differential equations for the film thickness, the volumetric flow rate, and the wall shear stress. This model 
is employable for moderate Reynolds numbers $(\mathrm{Re} \leqslant 100)$ and large Kapitza numbers $\left(\mathrm{Ka}=\left(\eta^{4} g\right) /\left(\rho \sigma^{3}\right)<8 \times 10^{-3}\right)$ and in this range, it is much better than other models as viscous effects of higher order were considered together with pressure corrections across the film thickness. Scheid, 2003 [16] developed a second-order WRIBL model which was capable to simulate the transition from two-dimensional to three-dimensional waves and the reaction of a film being subjected to nonhomogeneous heating.

Miyara, 1999 [17]; Miyara, 2000 [18]; Miyara, 2001 [19] investigated sinusoidally disturbed falling liquid films by solving the governing equations with the marker and cell algorithm for different Prandtl, Reynolds, Weber, and Froude numbers as well as for condensation. Groß, 2007 [20] and Soemers, 2008 [21] created a model for studying three-dimensional multiphase flow. The governing equations were discretised by employing the finite element method. In addition to the solution of the governing equations, the discretisation method offers an error estimation. Banerjee et al., 2004 [22], Fulgosi et al., 2003 [23], and Lakehal et al., 2003 [24] simulated turbulence and transport of scalar quantities across interfaces with direct numerical simulations for carbon dioxide in oceans, as an example. The authors found the turbulence development close to the interface to be similar to the turbulence development close to rigid walls but less anisotropic. Gambaryan-Roisman, 2007 [25] investigated the behaviour of a liquid film on a grooved surface with the Volume-of-Fluid method for interface tracking. The author considered thermocapillarity and also disruption of the film.

Lattice Boltzmann methods are a relatively new approach for simulating flow phenomena. Based on molecular gas dynamics, first models were presented in the late 1980s [26] (for a background, see [27]). Multiphase and multicomponent models, based on different approaches (e.g., see [2832]), have been mostly employed for describing drops or flow within porous media. To the best knowledge of the present authors, lattice Boltzmann methods have not yet been employed for simulating falling liquid film flows.

The aim of this study is to provide an approach for falling liquid film flow modelling by employing lattice Boltzmann methods. For this purpose, we assume isothermal two-phase flow that can be described by the model of Shan and Chen, 1993 [29]. Numerical tests are performed at low Reynolds numbers in order to investigate the ability of the model to predict the change in flow morphology from wave-less to laminar-wavy liquid film flow correctly. The paper is organised as follows. In Section 2 the numerical model is shown and details of the mathematical model, the geometry and mesh, the boundary and initial conditions, some preliminary tests and the parameters of the calculations are presented. In Section 3, the results of the calculations for disturbed and nondisturbed film flow are shown.

\section{Numerical Investigation}

For solving the hydrodynamics of a falling liquid film, the Shan-Chen model [29] has been employed, which is

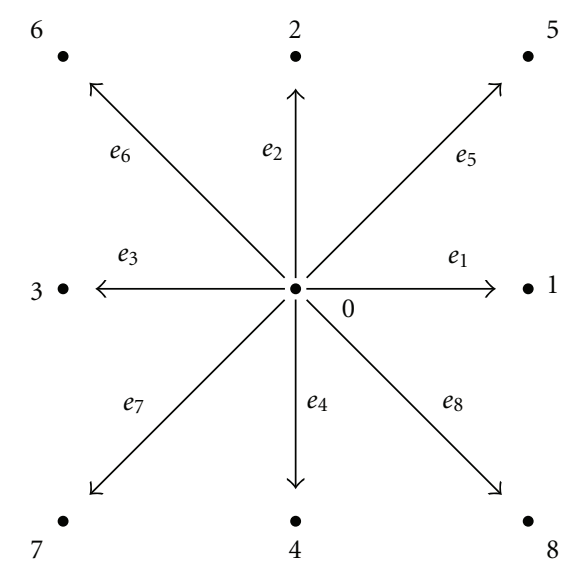

FIgURE 1: Velocity directions $i$ and velocity vectors $\mathbf{e}_{i}$ in D2Q9 lattice.

an isothermal lattice Boltzmann model for multiphase and multicomponent (with $\alpha$ components) flows.

2.1. Governing Equations. The lattics Boltzmann equation of the particle density distribution function of a component $\alpha$ reads

$$
f_{i}^{\alpha}\left(\mathbf{x}+\mathbf{e}_{i} \Delta t, t+\Delta t\right)-f_{i}^{\alpha}(\mathbf{x}, t)=\Omega^{\alpha},
$$

where $f_{i}^{\alpha}$ is the particle density distribution function in the velocity direction $i$ at a point in time $t$ and a time step $\Delta t$. The collision term $\Omega^{\alpha}$ represents the local interaction of the particles and is modelled with the well-known BGK approximation that leads to a linearisation of the collision term [33]. Thus,

$$
\Omega^{\alpha}=-\frac{1}{\tau^{\alpha}+0.5}\left(f_{i}^{\alpha}(\mathbf{x}, t)-f_{i}^{\alpha, \mathrm{eq}}(\mathbf{x}, t)\right),
$$

with $\tau^{\alpha}=v^{\alpha} c_{s}^{2}$ for the relaxation time, $v$ and $c_{s}$ for the kinematic viscosity and speed of sound, respectively, and $f_{i}^{\alpha, \text { eq }}$ for the equilibrium particle density distribution function (the exponent $\alpha$ will be dropped in the following, since only single-component flows with $\alpha=1$ are being investigated here), which can be derived from the Maxwell distribution by Taylor series and reads

$$
f_{i}^{\mathrm{eq}}(\mathbf{x}, t)=w_{i} \rho\left[1+3 \frac{\mathbf{e}_{i} \cdot \mathbf{u}^{\mathrm{eq}}}{c^{2}}+\frac{9}{2} \frac{\left(\mathbf{e}_{i} \cdot \mathbf{u}^{\mathrm{eq}}\right)^{2}}{c^{4}}-\frac{3}{2} \frac{\left(\mathbf{u}^{\mathrm{eq}}\right)^{2}}{c^{2}}\right] .
$$

Herein, $w_{i}$ is weighting factors of the specific velocity directions in a D2Q9 lattice (D2Q9 implies two space dimensions and nine velocity directions) (see Figure 1) with the values

$$
w_{i}= \begin{cases}\frac{4}{9}, & \text { for } i=0, \\ \frac{1}{9}, & \text { for } i=1, \ldots, 4 \\ \frac{1}{36}, & \text { for } i=5, \ldots, 8\end{cases}
$$


The vector $\mathbf{u}^{\mathrm{eq}}$ represents the macroscopic equilibrium velocity and the vectors $\mathbf{e}_{i}$ are the microscopic velocity vectors in the respective directions, that read

$$
\begin{array}{r}
\frac{\mathbf{e}_{i}}{c}=\left(\begin{array}{ccccccccc}
0 & 1 & 0 & -1 & 0 & 1 & -1 & -1 & 1 \\
0 & 0 & 1 & 0 & -1 & 1 & 1 & -1 & -1
\end{array}\right) \\
\text { for } i \in\{0,1, \ldots, 8\} .
\end{array}
$$

Herein, $c=1 \mathrm{lu} \mathrm{ts}^{-1}$ is the reference velocity in lattice units (lattice units can be converted to physical units either by nondimensional numbers or by conversion factors. E., g., for the length $\zeta_{l}=L_{\mathrm{P}} / L_{\mathrm{LB}}$, velocity $\zeta_{u}=u_{\mathrm{P}} / u_{\mathrm{LB}}$, and time $\left.\zeta_{t}=\zeta_{l} / \zeta_{u}\right)$. The variable $\rho$ is the mass density at a site $\mathbf{x}$ and can be expressed by the zeroth moment of the distribution function

$$
\rho(\mathbf{x}, t)=m \sum_{i=0}^{8} f_{i},
$$

with the molecular mass $m$. The equation for the macroscopic equilibrium velocity $\mathbf{u}^{\mathrm{eq}}$ consists of two parts. The first part depends on the particle density distribution function at the current time and the second part depends on the effect of external forces $\mathbf{F}$ that determine the velocity field. Thus,

$$
\mathbf{u}^{\mathrm{eq}}=\frac{m}{\rho} \sum_{i=0}^{8} \mathbf{e}_{i} f_{i}+\frac{\tau \mathbf{F}}{\rho}
$$

The vector of all forces $\mathbf{F}$ is the superposition of three forces, that is,

$$
\mathbf{F}=\mathbf{F}_{\mathrm{INT}}+\mathbf{F}_{\mathrm{FW}}+\mathbf{F}_{\mathrm{GR}},
$$

wherein $\mathbf{F}_{\mathrm{INT}}$ is the interphase force, $\mathbf{F}_{\mathrm{FW}}$ is the fluid-wall force and $\mathbf{F}_{\mathrm{GR}}$ is the gravitational force. The interphase force can be calculated with

$$
\mathbf{F}_{\mathrm{INT}}(\mathbf{x}, t)=-G \psi(\mathbf{x}, t) \sum_{i=1}^{8} w_{i} \psi\left(\mathbf{x}+\mathbf{e}_{i} \Delta t, t\right) \mathbf{e}_{i},
$$

where $G$ is the cohesion parameter and $\psi$ is the interaction potential that is defined by

$$
\begin{gathered}
\psi(\mathbf{x}, t)=\psi_{0} \exp \left(-\frac{\rho_{0}}{\rho}\right) \quad \text { with } \rho=\rho(\mathbf{x}, t), \\
\psi_{0}=4.0, \quad \rho_{0}=200 .
\end{gathered}
$$

The interphase force leads to the effects of phase separation and surface tension. From (9) and (10), the equation of state can be calculated with

$$
p=\frac{\rho}{3}+\frac{G}{6} \psi^{2}(\mathbf{x}, t),
$$

wherein the first term represents the ideal gas law and the second term represents a nonlinear part being shaped like the van-der-Waals equation.

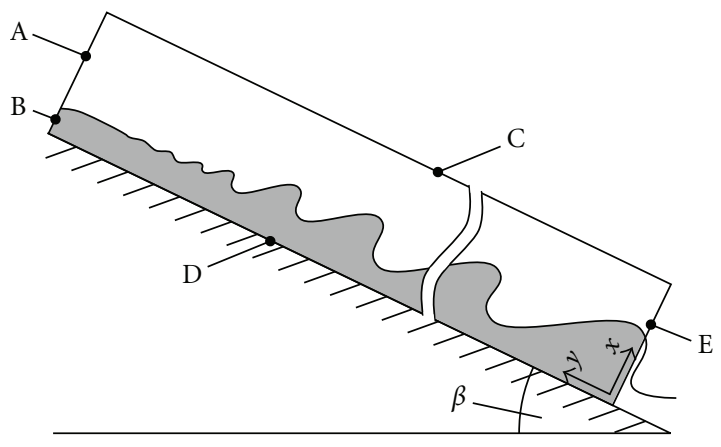

FIGURE 2: Geometry of the computational domain (black box); A: wall, B: liquid inlet velocity, C and D: walls, E: gradient-free outlet; $\beta$ : inclination angle against horizon.

The fluid-wall forces can be calculated by

$$
\mathbf{F}_{\mathrm{FW}}(\mathbf{x}, t)=-G_{\mathrm{ads}} \psi(\mathbf{x}, t) \sum_{i=1}^{8} w_{i} s\left(\mathbf{x}+\mathbf{e}_{i} \Delta t\right) \mathbf{e}_{i},
$$

wherein $G_{\text {ads }}$ is the adsorption parameter and the variable $s$

$$
s\left(\mathbf{x}+\mathbf{e}_{i} \Delta t\right)= \begin{cases}1, & \text { if } \mathbf{x}+\mathbf{e}_{i} \Delta t \text { is a wall node, } \\ 0, & \text { if } \mathbf{x}+\mathbf{e}_{i} \Delta t \text { is not a wall node }\end{cases}
$$

The interrelation of the cohesion parameter $G$ and the adsorption parameter $G_{\text {ads }}$ is represented by

$$
G_{\mathrm{ads}}=G \psi(\rho(\mathbf{x})),
$$

whereby the contact angle $\theta$ at the three-phase point can be correlated with the density $\rho[34]$ as

$$
\psi(\rho(\mathbf{x}))= \begin{cases}\psi\left(\rho_{\mathrm{F}}\right) & \text { for } \theta=0^{\circ}, \\ 0.5\left[\psi\left(\rho_{\mathrm{F}}\right)+\psi\left(\rho_{\mathrm{G}}\right)\right] & \text { for } \theta=90^{\circ}, \\ \psi\left(\rho_{\mathrm{G}}\right) & \text { for } \theta=180^{\circ} .\end{cases}
$$

The computation of the gravitational force can be performed with

$$
\mathbf{F}_{\mathrm{GR}}(\mathbf{x}, t)=\rho(\mathbf{x}, t) \mathbf{g},
$$

where $\mathbf{g}$ is the vector of the gravitational acceleration.

By using a Chapman-Enskog expansion (see, e.g., [35]), Shan and Chen, 1993 [29] have derived the macroscopic balancing equations at the limits of low disturbance frequency and long wave lengths. Some thermodynamic properties of the model have been additionally discussed by $\mathrm{He}$ and Doolen, 2002 [30] and Chen et al., 2007 [26].

2.2. Geometry and Mesh. A falling liquid film is investigated, flowing down an inclined wall with an angle $\beta$ against horizon, whereupon it is assumed that the film only develops along the wall. This implies that the domain can be reduced to two dimensions as shown in Figure 2. In this figure, also the boundary conditions are marked. More details about the boundary conditions are given in Section 2.3.

The applied discretisation scheme (D2Q9) can be used only for uniform lattices, wherein the spacing is limited by two dependencies as follows. 


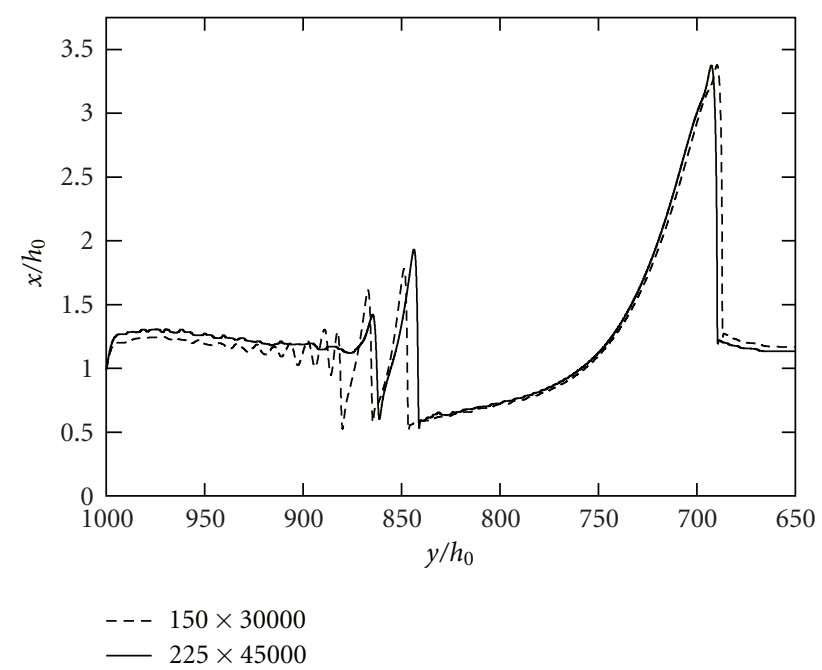

FIgURE 3: Grid independence test for $\operatorname{Re}=20$ without sinusoidal disturbances with the given resolutions at $t=0.2 \mathrm{~s}$.

(i) Necessary resolution of film thickness (Kuzmin and Derksen, 2011 [36] showed that the film thickness should be at least twice as thick as the interface thickness. Here, we have an interface thickness of five grid points.)

$$
\delta_{x} \lesssim \frac{1}{10} h_{0}, \quad h_{0}=1.0 \mathrm{lu} .
$$

(ii) For neglecting compressibility effects,

$$
\begin{aligned}
\mathrm{Ma} & =\frac{u}{c_{\mathrm{S}}} \leq 0.1 \\
& =\sqrt{3} u=\sqrt{3} \frac{\delta_{t}}{\delta_{x}} \approx \sqrt{3} \frac{\eta_{\mathrm{t}} \delta_{x}^{2}}{\delta_{x}}=\sqrt{3} \eta_{\mathrm{t}} \delta_{x} l \mathrm{u} \leq 0.1 .
\end{aligned}
$$

By virtue of

$$
u=\frac{\delta_{t}}{\delta_{x}}=\eta_{t} \delta_{x} \quad \text { with } \delta_{t} \propto \delta_{x}^{2} .
$$

Obviously, the demand for a small Mach number undergoes the demand for sufficient resolution of the film; thus, the critical space resolution is

$$
\delta_{x} \lesssim 0.1\left(\sqrt{3} \eta_{\mathrm{t}} \mathrm{lu}\right)^{-1} \quad \text { with } \eta_{\mathrm{t}}=2.0
$$

The extent of the computational domain depends upon the length of film under consideration and upon the prospective wave magnitude. The dimensions in both coordinate directions are given as a multiple of the inlet film thickness $h_{0}$, wherein the length is $L=1000 h_{0}$ and the width is $H=5 h_{0}$. This results in a mesh of $30000 \times 150$ nodes, since the film thickness is resolved by 30 nodes. The grid independence test has been performed and the result for $\mathrm{Re}=20$ is shown in Figure 3.

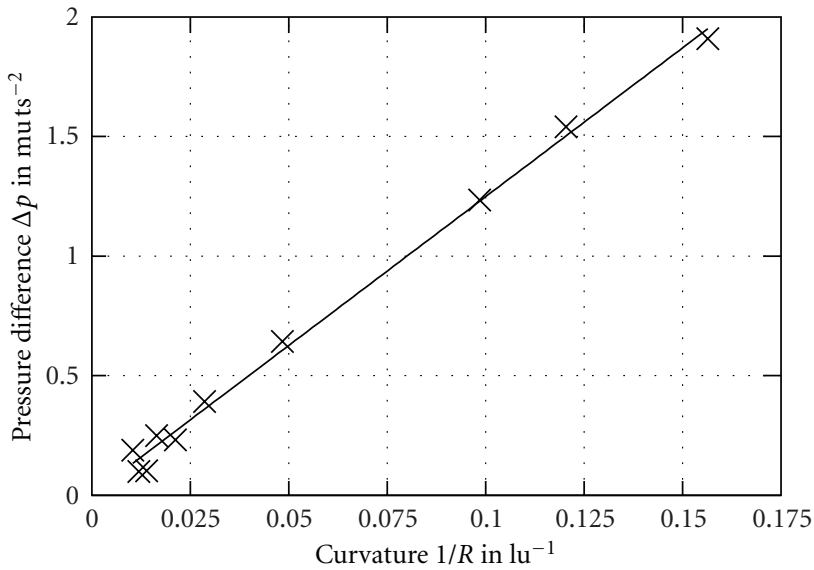

FIgURE 4: Evaluation of the pressure difference depending on curvature of the drop.

2.3. Boundary and Initial Conditions. The boundary conditions are shown in Figure 2. There are three solid wall boundaries (A, C, D) modelled by bounce-back, a velocity inlet (B), and a zero gradient outlet. The velocity given in $B$ is constant in space but may be varied in time with

$$
u_{y}=u_{0}[1+\varepsilon \sin (2 \pi F t)] \text {, }
$$

where $u_{0}$ is the initial liquid velocity, $\varepsilon$ and $F$ are the relative amplitude and frequency of the disturbance, respectively, whereas the velocity component in $x$ direction remains $u_{x}=$ 0 . The missing incoming distribution functions are calculated by using the Zou and He boundary condition Zou and He, 1997 [37].

The outlet (E) was assumed to be free of gradients, which is realised by setting all $f_{i}$ at the boundary node equal to the corresponding value of $f_{i}$ at the neighbouring node in the fluid.

For initialising the domain, the film was supplied with a constant thickness $h_{0}$ and a uniform velocity $\mathbf{u}_{0}=\left(0, u_{0}\right)^{T}$. The gas was assumed to be at rest.

2.4. Implementation and Validation of the Numerical Model. The previously described model has been implemented in a self-written serial C++-program, which has been validated with single- and two-phase benchmark tests. Computations have been conducted for Poiseuille flow and flow around a cylinder which showed an excellent performance of the code for single-phase flows. The two-phase benchmarks were the transition from a liquid square to a circle and the following determination of the surface tension as well as the investigation of the contact angle formation at the threephase points [38].

The square-to-circle computations have been performed by defining squares of different sizes being transformed to circles, whose radii have been measured. The pressure difference between inside and outside of the drop has been also determined. In Figure 4 , the pressure difference $\Delta p$ is plotted versus the curvature $1 / R$. It is shown that the results can be fitted by a linear regression line and that the deviation 
is relatively high for small curvatures, which occurs due to low pressure differences. From the regression line, the surface tension can be calculated to be $\sigma=(12.45 \pm 0.32) \mathrm{mu} \mathrm{lu} \mathrm{ts}^{-2}$.

2.5. Test Series and Parameters. The characteristic velocity $u_{0, \mathrm{P}}$ and the initial film thickness $h_{0, \mathrm{P}}$ may be calculated with

$$
\begin{gathered}
u_{0, \mathrm{P}}=\sqrt[3]{\frac{1}{3} \operatorname{Re}^{2} v_{\mathrm{F}, \mathrm{P}} g_{\mathrm{P}} \sin \beta}, \\
h_{0, \mathrm{P}}=\sqrt[3]{3 \operatorname{Re} \frac{v_{\mathrm{F}, \mathrm{P}}^{2}}{g_{\mathrm{P}} \sin \beta}},
\end{gathered}
$$

respectively, following Nusselt, 1916, [39]. Herein, Re is the Reynolds number defined as

$$
\operatorname{Re}=\frac{u_{0, \mathrm{P}} h_{0, \mathrm{P}}}{v_{\mathrm{F}, \mathrm{P}}},
$$

where $\nu_{\mathrm{F}, \mathrm{P}}$ is the kinematic viscosity of the film and $g_{\mathrm{P}}$ is the magnitude of the gravitational acceleration with an angle $\beta$ against horizon. The Kapitza number,

$$
\mathrm{Ka}=\frac{\mu_{\mathrm{F}}^{4} g}{\rho_{\mathrm{F}} \sigma^{3}},
$$

a dimensionless number, only depending on properties of the fluid and on gravitational acceleration, cannot be predefined in this model, since both fluid densities and the surface tension are coupled by the cohesion parameter $G$ and the viscosity is linked to the Reynolds number.

By employing the present implementation, the following test cases have been investigated for a vertical wall $\left(\beta=90^{\circ}\right.$ as follows):

(i) non-disturbed flow at $\operatorname{Re} \in\{5,8,20\}$ (free transition)

(ii) sinusoidally disturbed flow at $\mathrm{Re}=20.1$. 1.

The parameters for the computations are shown in Table

\section{Results and Discussion}

3.1. Nondisturbed Film. The hydrodynamics of falling liquid films without artificial disturbance at the inlet have been studied for three different Reynolds numbers representing specific changes in the flow morphology according to Gross et al., 2009. [5].

In the following sections, the wave shapes depending on time and space are shown as well as the velocity fields and the characteristic wave velocities.

3.1.1. Evolution of the Interface. Figures 5 to 7 illustrate the evolution of the interface with time for different Reynolds numbers. Every figure is composed of ten subfigures in which the film thickness is plotted versus the strongly compressed

\begin{tabular}{|c|c|c|c|c|}
\hline & $\operatorname{Re}=5$ & $\mathrm{Re}=8$ & $\operatorname{Re}=20$ & $\mathrm{Re}=20.1$ \\
\hline$h_{0, \mathrm{P}} / \mathrm{mm}$ & 0.115 & 0.135 & 0.183 & 0.184 \\
\hline$u_{0, \mathrm{P}} /(\mathrm{mm} / \mathrm{s})$ & 43.50 & 59.51 & 109.5 & 109.9 \\
\hline$H_{\mathrm{P}} / \mathrm{mm}$ & 0.578 & 0.676 & 0.917 & 0.919 \\
\hline$L_{\mathrm{P}} / \mathrm{mm}$ & 115.5 & 135.2 & 183.3 & 183.8 \\
\hline$t_{0, \mathrm{P}} / \mathrm{s}$ & 2.655 & 2.272 & 1.674 & 1.672 \\
\hline$\varepsilon$ & 0 & 0 & 0 & 0.05 \\
\hline$F /(1 / s)$ & 0 & 0 & 0 & 45 \\
\hline$g_{\mathrm{P}} /\left(\mathrm{m} / \mathrm{s}^{2}\right)$ & 2.452 & 2.452 & 4.660 & 4.415 \\
\hline $\mathrm{Ka} / 10^{-3}$ & 28.58 & 3.544 & 0.050 & 0.049 \\
\hline$\beta 1^{\circ}$ & \multicolumn{4}{|c|}{90.0} \\
\hline$v_{\mathrm{F}, \mathrm{P}} /\left(\mathrm{m}^{2} / \mathrm{s}\right)$ & \multicolumn{4}{|c|}{$1.004809 \times 10^{-6}$} \\
\hline G & \multicolumn{4}{|c|}{-120.0} \\
\hline$G_{\text {ads }}$ & \multicolumn{4}{|c|}{-187.16} \\
\hline$\rho_{\mathrm{F}} /\left(\mathrm{mu} / \mathrm{lu}^{2}\right)$ & \multicolumn{4}{|c|}{528.8} \\
\hline$\rho_{\mathrm{G}} /\left(\mathrm{mu} / \mathrm{lu}^{2}\right)$ & \multicolumn{4}{|c|}{86.4} \\
\hline$\sigma /\left(\mathrm{mulu} / \mathrm{ts}^{2}\right)$ & \multicolumn{4}{|c|}{14.33} \\
\hline
\end{tabular}
axial position in the film. The subfigures are shown in a subsequent order and have a specific value for a variable $k$,
TABLE 1: Parameters for computation.

whereupon the respective real time is the product of $k$ and a characteristic time step. Every last subfigure $(k=9)$, that is, the uppermost one, shows the film interface at time

$$
\frac{t}{t_{0, \mathrm{P}}}=\frac{t u_{0, \mathrm{P}}}{L_{\mathrm{D}} h_{0, \mathrm{P}}}=2 \quad \text { with } L_{\mathrm{D}}=\frac{L_{\mathrm{P}}}{h_{0, \mathrm{P}}}=1000,
$$

which implies that a fluid particle with the velocity $u_{0, \mathrm{P}}$ has crossed the complete domain twice. This time corresponds to the time in discrete units of $t=900000$ ts.

The wave evolution for $\operatorname{Re}=5$ is shown in Figure 5. It can be observed that for $k=1\left(t_{\mathrm{P}}=0.590 \mathrm{~s}\right)$, first waves occur from $y / h_{0} \lesssim 800$ on with a small amplitude. In the next following points in time, the position of wave initiation flows downstream, and it stabilises at $y / h_{0} \approx 200$ for times greater than $t_{\mathrm{P}} \gtrsim 2.950 \mathrm{~s}(k \gtrsim 5)$. The waves occurring here are limited in their amplitude to approximately half of the initial film thickness $h_{0}$. The observations of these calculations correspond qualitatively good to experimental data obtained by Gross et al., 2009, [5], who found that a water film in a vertical tube has a first transition at $\mathrm{Re} \approx 6$ from a laminar wave-less shape to small waves.

In Figure 6, the evolution of the film surface is shown for $\mathrm{Re}=8$. Contrary to $\mathrm{Re}=5$, a first solitary wave occurs flowing downstream faster than the other waves. This wave has its origin in the starting of the calculation. During the whole computation process no new solitary wave was observed for this Reynolds number. It can be seen that small waves appear only behind the solitary wave and that the beginning of the wave production is also moving downstream. The small wave amplitudes are higher than the ones occurring at $\mathrm{Re}=5$. According to Gross et al., 2009, [5] a water film has so-called "small waves" at $\operatorname{Re}=8$, which also can be observed here.

The wave evolution for $\operatorname{Re}=20$ is shown in Figure 7 . Similar to $\operatorname{Re}=8$, a first solitary wave establishes whose magnitude increases until the top of the wave reaches the 


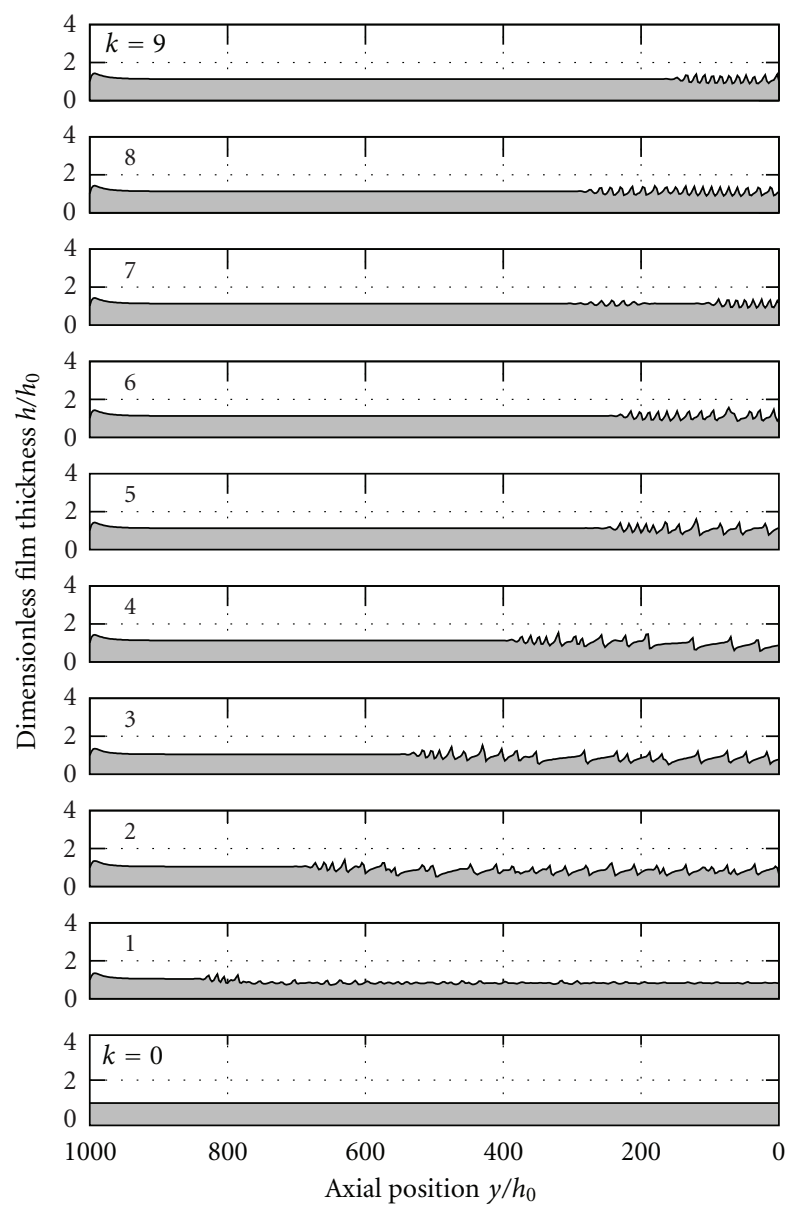

FIgURE 5: Evolution of the interface with $\mathrm{Re}=5$ at different points in time; the subfigures 0 to 9 show the evolution for increasing time until $t_{9}=2 t_{0, \mathrm{P}}=5.310 \mathrm{~s}$, whereby $t_{k}=k \cdot 0.590 \mathrm{~s}$, with $k \in\{0,1,2, \ldots, 9\}$.

upper side of the domain $\left(k=1\right.$ at $\left.y / h_{0} \approx 340\right)$. This wave and also the waves further downstream appear due to the starting of the computation. Upstream the solitary wave a small wave producing region establishes whose position remains the same during the whole computation period, but with increasing length from $\Delta\left(y / h_{0}\right) \approx 150$ in $k=1$ to $\Delta\left(y / h_{0}\right) \approx 400$ in $k \approx 4$. From the wave producing region, waves are released whose magnitude is about twice the magnitude of the small waves, and some of them grow to more than $h / h_{0}=2$. Gross et al., 2009 [5] observed waves with increased magnitude and the occurrence of some bow waves (see Figure $7, k=9$ at $y / h_{0} \approx 410$ and Figure 9).

The figures shown here demonstrate that it is possible to model the hydrodynamics of falling liquid films by lattice Boltzmann methods, but it should be mentioned that the direct comparison of these results with experimental data is difficult, due to the limitations of the model; the fluid densities are coupled with the surface tension by the cohesion parameter $G$ which reduces the degrees of freedom for realistic cases.

3.1.2. Velocity Field. For better insight, two sections of the flow field are shown in Figures 8 and 9 for $\operatorname{Re}=20$ at $k=9$

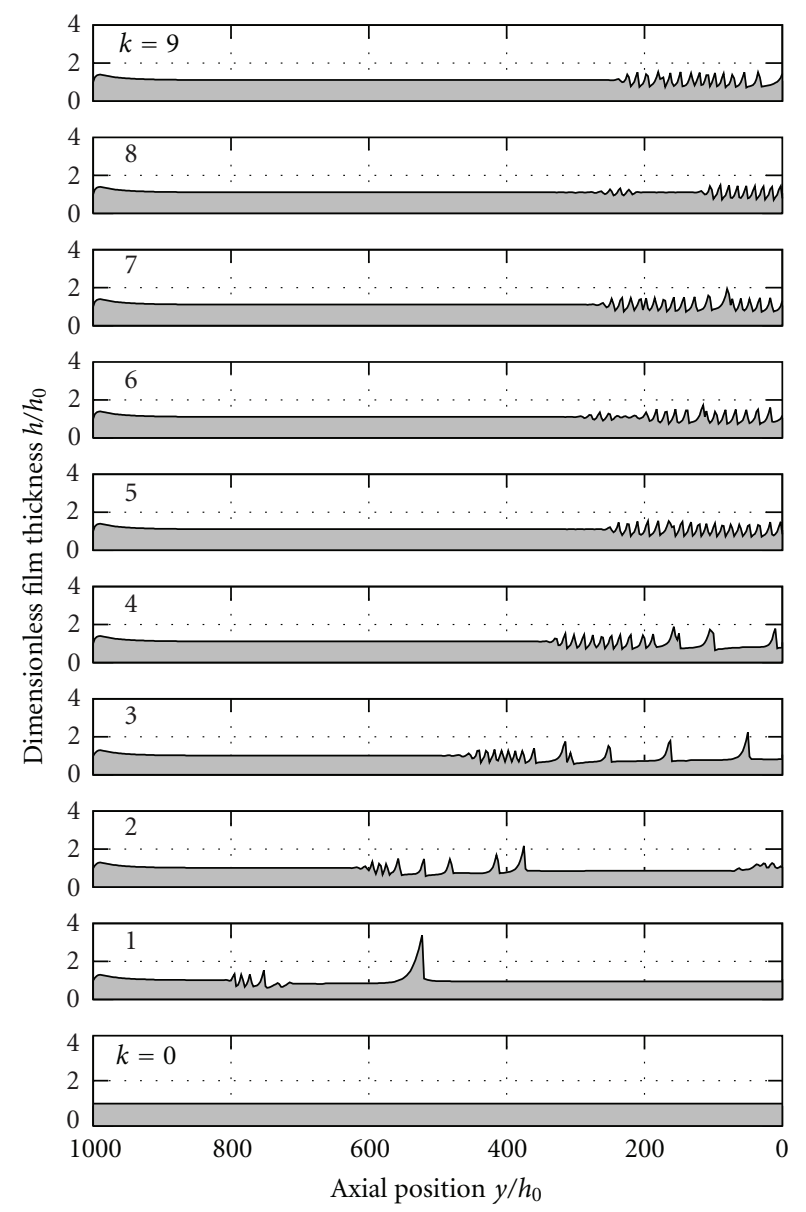

FIGURE 6: Evolution of the interface with $\mathrm{Re}=8$ at different points in time; the subfigures 0 to 9 show the evolution for increasing time until $t_{9}=2 t_{0, \mathrm{P}}=5.544 \mathrm{~s}$, whereby $t_{k}=k \cdot 0.504 \mathrm{~s}$, with $k \in\{0,1,2, \ldots, 9\}$.

$(t=3.348 \mathrm{~s})$. In both figures, the film (grey) is indicated and the velocity field is represented by arrows. Similar to the previously shown figures, the coordinate in flow direction is strongly compressed.

Figure 8 shows a section of the transition region from laminar wave-less to laminar wavy film. There are sinusoidal waves with a wavelength of approximately $10 h_{0}$ but with increasing amplitude, which corresponds to the experimental findings, where initially small waves occur whose amplitude increases with time. The velocity field shows a cocurrent motion of film and gas with the gas velocity being higher. Additionally, two effects can be seen. First, there are large velocity vectors directing in the gas phase and second, the film seems to flow towards the wall $\left(x / h_{0}=0\right)$. Both effects appear to be due to the interphase and fluid-wall forces modelled by interaction potentials. These spurious currents have been previously described by Chen et al., 2007 [26], and Sukop and Thorne jr. 2007 [34] who found out that the currents at the interface do not represent a mass transfer through the interface. The magnitude of the largest parasitic currents at the interface is approximately $1 \mathrm{lu} / \mathrm{ts}$ whilst the gas bulk velocity is approximately $0.1 \mathrm{lu} / \mathrm{ts}$. 


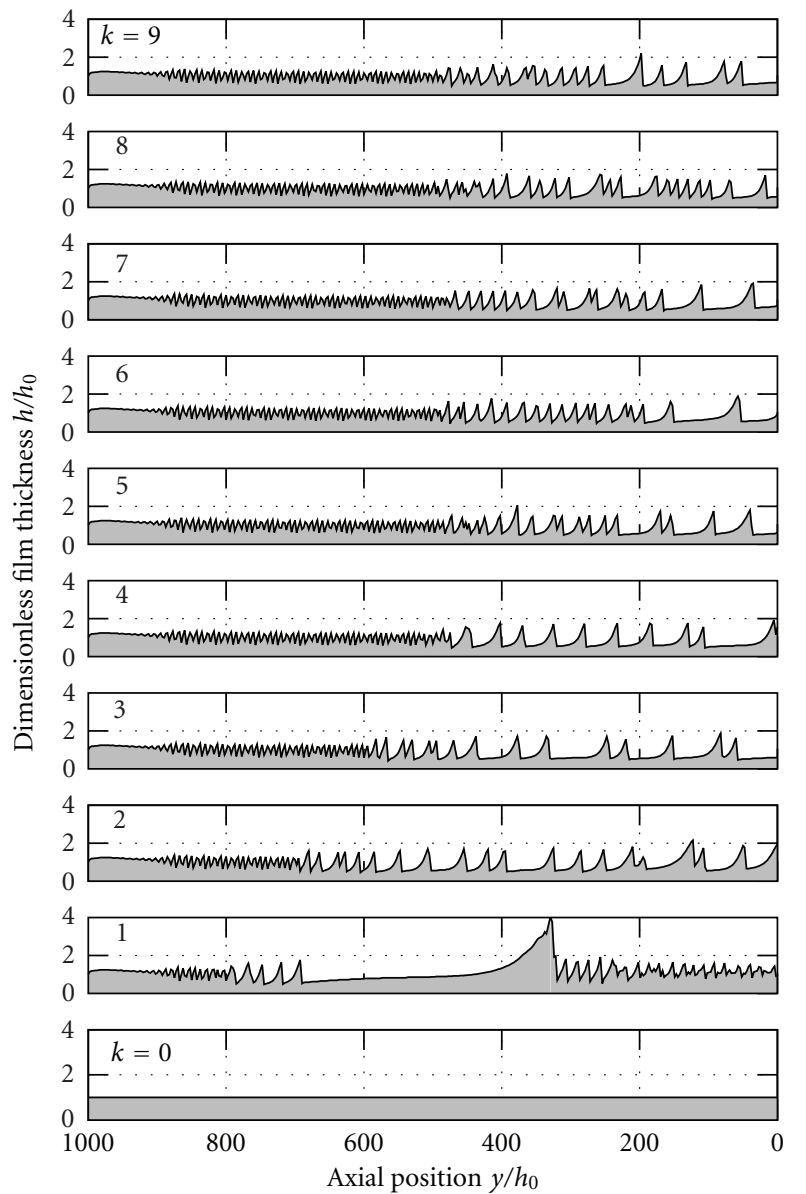

FIGURE 7: Evolution of the interface with $\mathrm{Re}=20$ at different points in time; the subfigures 0 to 9 show the evolution for increasing time until $t_{9}=2 t_{0, \mathrm{P}}=3.348 \mathrm{~s}$, whereby $t_{k}=k \cdot 0.372 \mathrm{~s}$, with $k \in\{0,1,2, \ldots, 9\}$.

In Figure 9, a wave with a bow wave in front of it is shown. Gross et al., 2009 [5] have discovered that water films exhibit bow waves for $\mathrm{Re}=20$ which can also be seen here. Obviously, both of these waves have a length of approximately $25 h_{0}$ and a much larger mightiness than the waves shown in Figure 8.

3.1.3. Wave Velocity. Both the wave velocity of a solitary wave and the velocity of a wave producing region can be evaluated by measuring wave positions at two consecutive points in time. In Table 2, the wave velocities are presented for the simulations being performed here. Good qualitatively agreement is obtained by comparing the results with experimental data shown by Philipp et al., 2006 [40] who reported an approximate wave velocity of $400 \mathrm{~mm} / \mathrm{s}$ for $\mathrm{Re}=20$. For lower Reynolds numbers no experimental data are available.

It can be seen in Table 2 that with increasing Reynolds number, the solitary wave velocity also increases but the velocity of the wave producing region almost remains constant, and for $\mathrm{Re}=20$ it is approximately zero.

3.2. Sinusoidally Disturbed Film. Apart from falling liquid films with a natural transition to wavy interface,

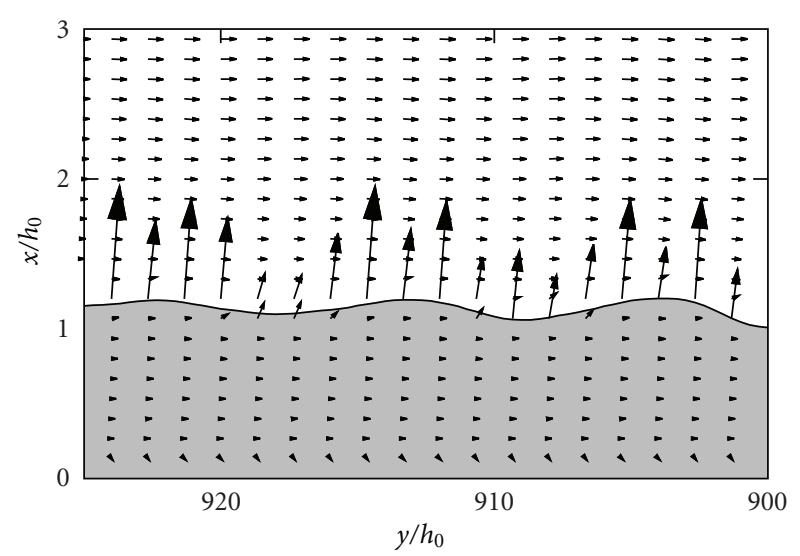

FIgURE 8: Section of the velocity field for $\operatorname{Re}=20$ (Figure $7, k=9$, $t=3.348 \mathrm{~s})$

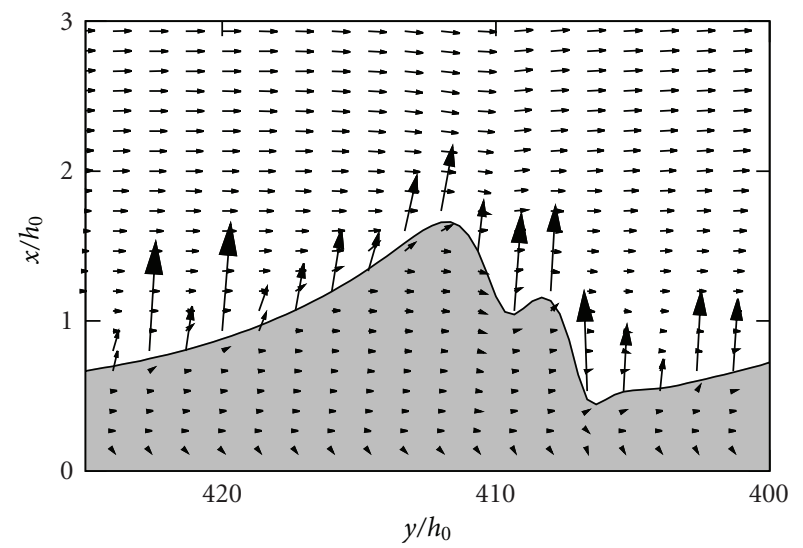

FIGURE 9: Section of the velocity field for $\operatorname{Re}=20$ (Figure $7, k=9$, $t=3.348 \mathrm{~s})$.

TABLE 2: Wave velocity of solitary waves (solitary w.) and beginning wave production (b. w. production).

\begin{tabular}{lcc}
\hline $\operatorname{Re}$ & \multicolumn{2}{c}{$u_{\text {wave }}$ in $\mathrm{mm} / \mathrm{s}$} \\
\hline 5 & Solitary w. & b. w. production \\
8 & - & 32.59 \\
20 & 126.86 & 42.74 \\
\hline
\end{tabular}

computations have been performed with a sinusoidally disturbed film, and the results are compared to those obtained by Gao et al., 2003 [41] with a volume of fluid approach and Navier-Stokes equations.

In Figure 10, the results of the present computations are presented and also those from literature. It can be seen that both approaches show large waves with smaller ones in-between. However, there is only a qualitative agreement between both. The reason for this can be found in the fact that the Shan-Chen model allows only limited variation of the Weber number (We $=\rho h_{0} u^{2} / \sigma$ ), and hence, the Weber number in our case is We $=2.4$ whilst Gao et al., 2003 [41] have a value of $\mathrm{We}=0.24$. 


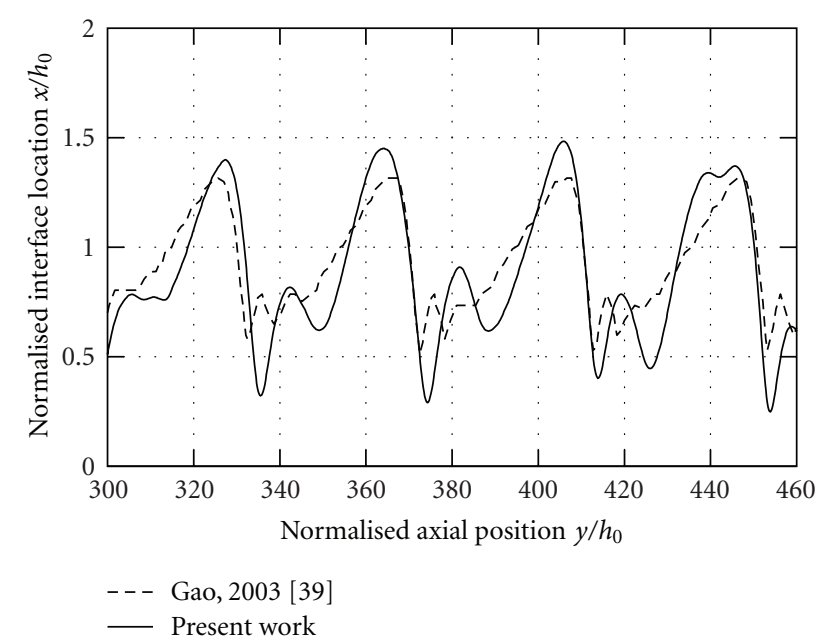

Figure 10: Comparison of the present results for $\mathrm{Re}=20.1, F=$ $45 \mathrm{~s}^{-1}$ and $\varepsilon=0.05$ with Gao et al., 2003 [41].

\section{Conclusion}

In the present investigation, falling liquid films on a vertical plane have been modelled with the Shan-Chen model, which is an isothermal multiphase and multicomponent lattice Boltzmann model based on interaction potentials. The implementation of the model has been validated against various single- and two-phase flow problems and proved its validity.

The simulation of falling liquid films has been conducted for cases with Reynolds numbers of $\operatorname{Re} \in\{5,8,20\}$ which showed that the employed Shan-Chen model is capable to simulate falling liquid film flows and that there is a good qualitative agreement with experimental data. Characteristic flow morphologies have been calculated for their respective Reynolds numbers. Direct comparison of the wave profiles is difficult, since limitations of the Shan-Chen model prevent that the Kapitza number can be freely chosen.

To conclude, it can be stated that the lattice Boltzmann methods are capable to simulate falling liquid films but the constraints given by the Shan-Chen model limit their generality. The investigation of liquid film flow with heat transfer, larger Reynolds numbers, and other geometrical parameters will be conducted in the future.

\section{Acknowledgment}

The authors greatly acknowledge the valuable comments and suggestions given by the unknown reviewers.

\section{References}

[1] P. L. Kapitza and S. P. Kapitza, "Wave flow of thin layers of a viscous fluid," Zhurnal Eksperimentalnoi I Theoreticheskoi Fiziki, vol. 19, pp. 105-120, 1949.

[2] P. Adomeit and U. Renz, "Deposition of fine particles from a turbulent liquid flow: experiments and numerical predictions," Chemical Engineering Science, vol. 31, pp. 3491-3503, 1996.
[3] P. Adomeit and U. Renz, "Hydrodynamics of three-dimensional waves in laminar falling films," International Journal of Multiphase Flow, vol. 26, no. 7, pp. 1183-1208, 2000.

[4] C. D. Park and T. Nosoko, "Three-dimensional wave dynamics on a falling film and associated mass transfer," AIChE Journal, vol. 49, no. 11, pp. 2715-2727, 2003.

[5] U. Gross, T. Storch, C. Philipp, and A. Doeg, "Wave frequency of falling liquid films and the effect on reflux condensation in vertical tubes," International Journal of Multiphase Flow, vol. 35, no. 4, pp. 398-409, 2009.

[6] C.-S. Yih, "Stability of parallel laminar flow with a free surface," Quarterly of Applied Mathematics, vol. 13, pp. 623-628, 1955.

[7] C.-S. Yih, "Stability of liquid flow down an inclined plane," Physics of Fluids, vol. 6, no. 3, pp. 321-334, 1963.

[8] T. B. Benjamin, "Wave formation in laminar flow down an inclined plane," The Journal of Fluid Mechanics, vol. 2, no. 6, pp. 554-574, 1957.

[9] D. J. Benney, "Long waves on liquid films," Journal of Mathematical Physics, vol. 45, pp. 150-155, 1966.

[10] A. Oron, S. H. Davis, and S. G. Bankoff, "Long-scale evolution of thin liquid films," Reviews of Modern Physics, vol. 69, no. 3, pp. 931-980, 1997.

[11] V. Y. Shkadov, "Wave flow regimes of a thin layer of viscous fluid subject to gravity," MekhanIka ZhIdkostI i Gaza, vol. 2, no. 1, pp. 43-51, 1967.

[12] C. Ruyer-Quil and P. Manneville, "Modeling film flows down inclined planes," European Physical Journal B, vol. 6, no. 2, pp. 277-292, 1998.

[13] C. Ruyer-Quil and P. Manneville, "Improved modeling of flows down inclined planes," European Physical Journal B, vol. 15, no. 2, pp. 357-369, 2000.

[14] C. Ruyer-Quil and P. Manneville, "Further accuracy and convergence results on the modeling of flows down inclined planes by weighted-residual approximations," Physics of Fluids, vol. 14, no. 1, pp. 170-183, 2002.

[15] L. T. Nguyen and V. Balakotaiah, "Modeling and experimental studies of wave evolution on free falling viscous films," Physics of Fluids, vol. 12, no. 9, pp. 2236-2256, 2000.

[16] B. Scheid, Evolution and stability of falling liquid films with thermocapillary effects [Ph.D. thesis], Université Libre de Bruxelles, 2003.

[17] A. Miyara, "Numerical analysis on flow dynamics and heat transfer of falling liquid films with interfacial waves," Heat and Mass Transfer, vol. 35, no. 4, pp. 298-306, 1999.

[18] A. Miyara, "Numerical simulation of wavy liquid film flowing down on a vertical wall and an inclined wall," International Journal of Thermal Sciences, vol. 39, no. 9-11, pp. 1015-1027, 2000.

[19] A. Miyara, "Flow dynamics and heat transfer of wavy condensate film," Journal of Heat Transfer, vol. 123, no. 3, pp. 492-500, 2001.

[20] S. Groß, Numerical methods for three-dimensional incompressible two-phase flow problems [Ph.D. thesis], RWTH Aachen, Aachen, Germany, 2007.

[21] M. Soemers, Numerical methods for the solution of a threedimensional anisotropic inverse heat conducting problem [Ph.D. thesis], RWTH Aachen, Aachen, Germany, 2008.

[22] S. Banerjee, D. Lakehal, and M. Fulgosi, "Surface divergence models for scalar exchange between turbulent streams," International Journal of Multiphase Flow, vol. 30, no. 7-8, pp. 963-977, 2004. 
[23] M. Fulgosi, D. Lakehal, S. Banerjee, and V. De Angelis, "Direct numerical simulation of turbulence in a sheared air-water flow with a deformable interface," Journal of Fluid Mechanics, vol. 482, pp. 319-345, 2003.

[24] D. Lakehal, M. Fulgosi, G. Yadigaroglu, and S. Banerjee, "Direct numerical simulation of turbulent heat transfer across a mobile, sheared gas-liquid interface," Journal of Heat Transfer, vol. 125, no. 6, pp. 1129-1139, 2003.

[25] T. Gambaryan-Roisman, Thin liquid films onstructured walls: hydrodynamics, heat transfer and stabilityred walls: hydrodynamics, heat transfer and stability [Habilitation thesis], Technische Universität Darmstadt, Darmstadt, Germany, 2007.

[26] S. Chen, X. He, and L.-S. Luo, "Computational methods for multiphase flow," in Lattice Boltzmann Models for Multiphase Flow, A. Prosperetti and G. Tryggvason, Eds., chapter 6, pp. 157-192, Cambridge University Press, Cambridge, UK, 2007.

[27] A. A. Mohamad, Lattice Boltzmann Method, Fundamentals and Engineering Applications with Computer Codes, Springer, London, UK, 2011.

[28] D. H. Rothman and J. M. Keller, "Immiscible cellularautomaton fluids," Journal of Statistical Physics, vol. 52, no. 3-4, pp. 1119-1127, 1988.

[29] X. Shan and H. Chen, "Lattice Boltzmann model for simulating flows with multiple phases and components," Physical Review E, vol. 47, no. 3, pp. 1815-1819, 1993.

[30] X. He and G. D. Doolen, "Thermodynamic foundations of kinetic theory and lattice Boltzmann models for multiphase flows," Journal of Statistical Physics, vol. 107, no. 1-2, pp. 309-328, 2002.

[31] T. Inamuro, T. Ogata, S. Tajima, and N. Konishi, "A lattice Boltzmann method for incompressible two-phase flows with large density differences," Journal of Computational Physics, vol. 198, no. 2, pp. 628-644, 2004.

[32] T. Lee and P. F. Fischer, "Eliminating parasitic currents in the lattice Boltzmann equation method for nonideal gases," Physical Review E, vol. 74, Article ID 046709, 7 pages, 2006.

[33] P. L. Bhatnagar, E. P. Gross, and M. Krook, "A model for collision processes in gases. I. Small amplitude processes in charged and neutral one-component systems," Physical Review, vol. 94, no. 3, pp. 511-525, 1954.

[34] M. C. Sukop and D. T. Thorne Jr., Lattice Boltzmann Modeling: An Introduction for Geoscientists and Engineers, Springer, Heidelberg, Germany, 2007.

[35] S. Chapman and T. G. Cowling, The Mathematical Theory of Non-Uniform Gases, Cambridge University Press, Cambridge, UK, 3rd edition, 1970.

[36] A. Kuzmin and J. Derksen, "The simulations of Bretherton phenomena in microchannels," in Proceedings of the 8th International Conference on Mesoscopic Methods in Engineering and Science (ICMMES '11), Lyon, France, 2011.

[37] Q. Zou and X. He, "On pressure and velocity boundary conditions for the lattice Boltzmann BGK model," Physics of Fluids, vol. 9, no. 6, pp. 1591-1596, 1997.

[38] A. Hantsch, Numerische Berechnung des Wärmeüberganges in einem fallenden Flüssigkeitsfilm mittels Gitter-BoltzmannMethoden [M.S. thesis], TU Bergakademie Freiberg, Freiberg, Germany, 2009.

[39] W. Nusselt, "Die Oberächenkondensation des Wasserdampfes," Zeitschrift des Vereines Deutscher Ingenieure, vol. 60, pp. 541546, 569-541-546, 575, 1916.
[40] C. Philipp, A. Doeg, S. Kufaas Tellefsen, and U. Gross, "Wave characterstics of liquid films-correlation of heat transfer data with visual observations," in Proceedings of the 13th International Heat Transfer Conference, 2006.

[41] D. Gao, N. B. Morley, and V. Dhir, "Numerical simulation of wavy falling film flow using VOF method," Journal of Computational Physics, vol. 192, no. 2, pp. 624-642, 2003. 

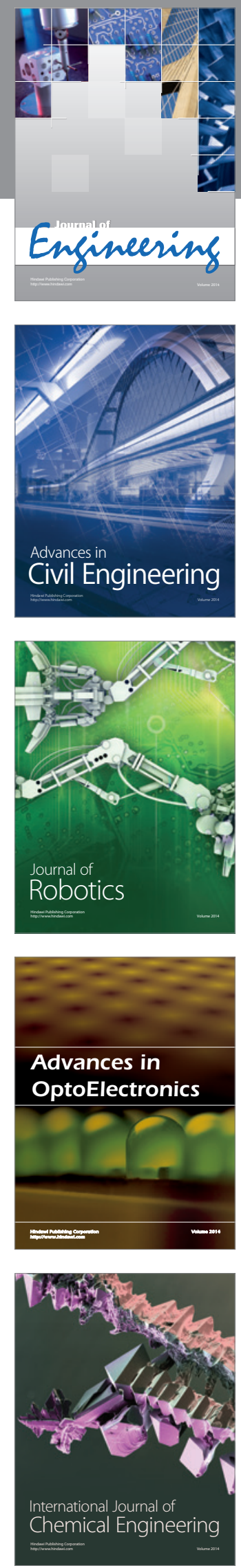

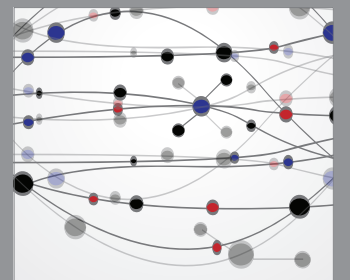

The Scientific World Journal
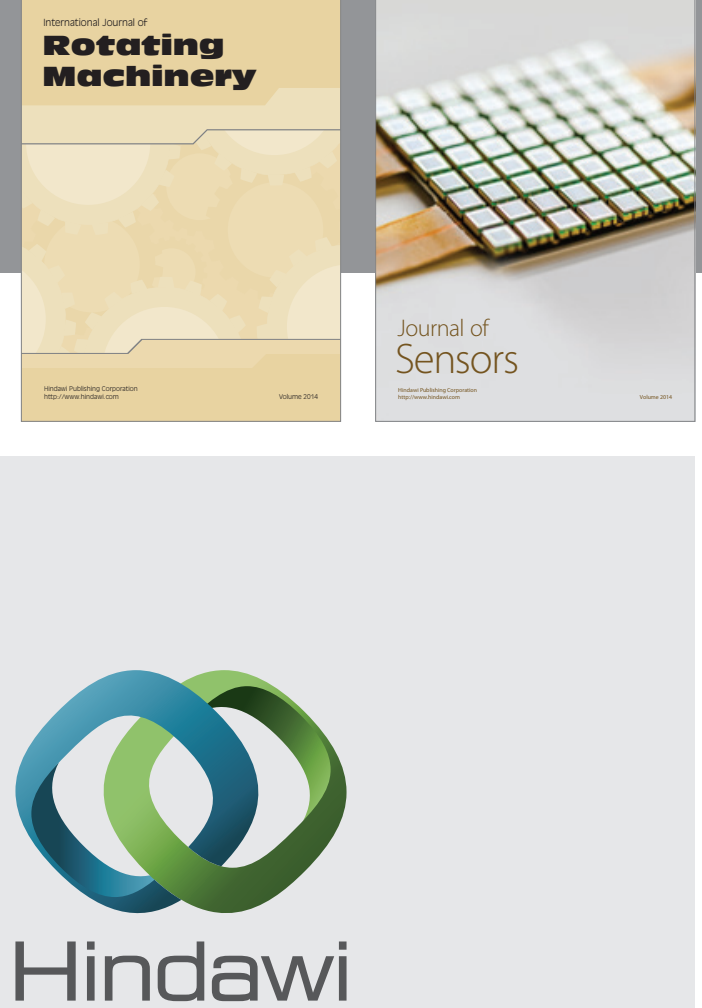

Submit your manuscripts at http://www.hindawi.com
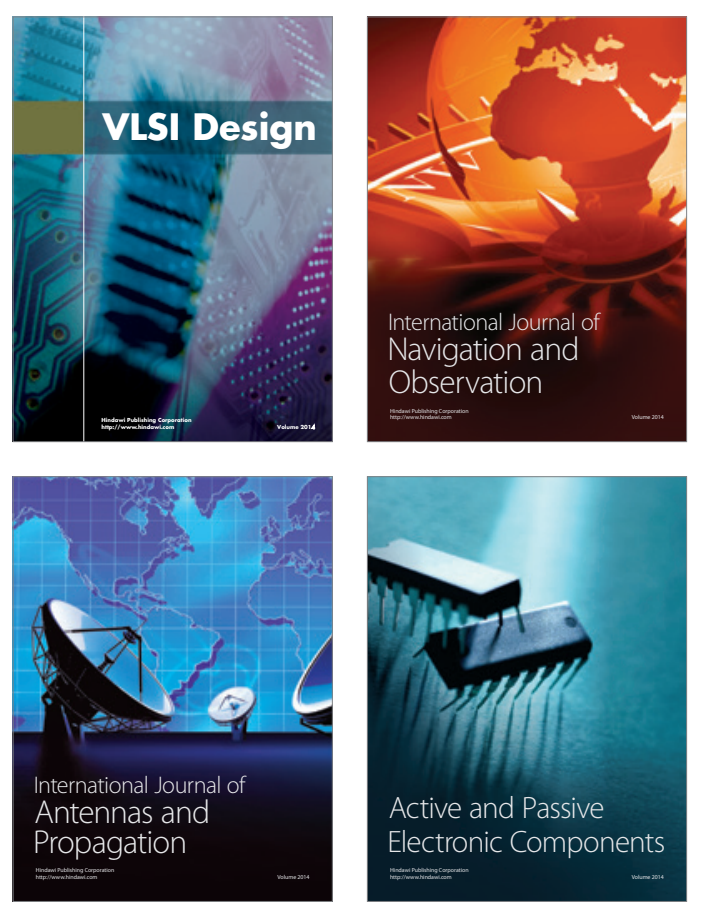
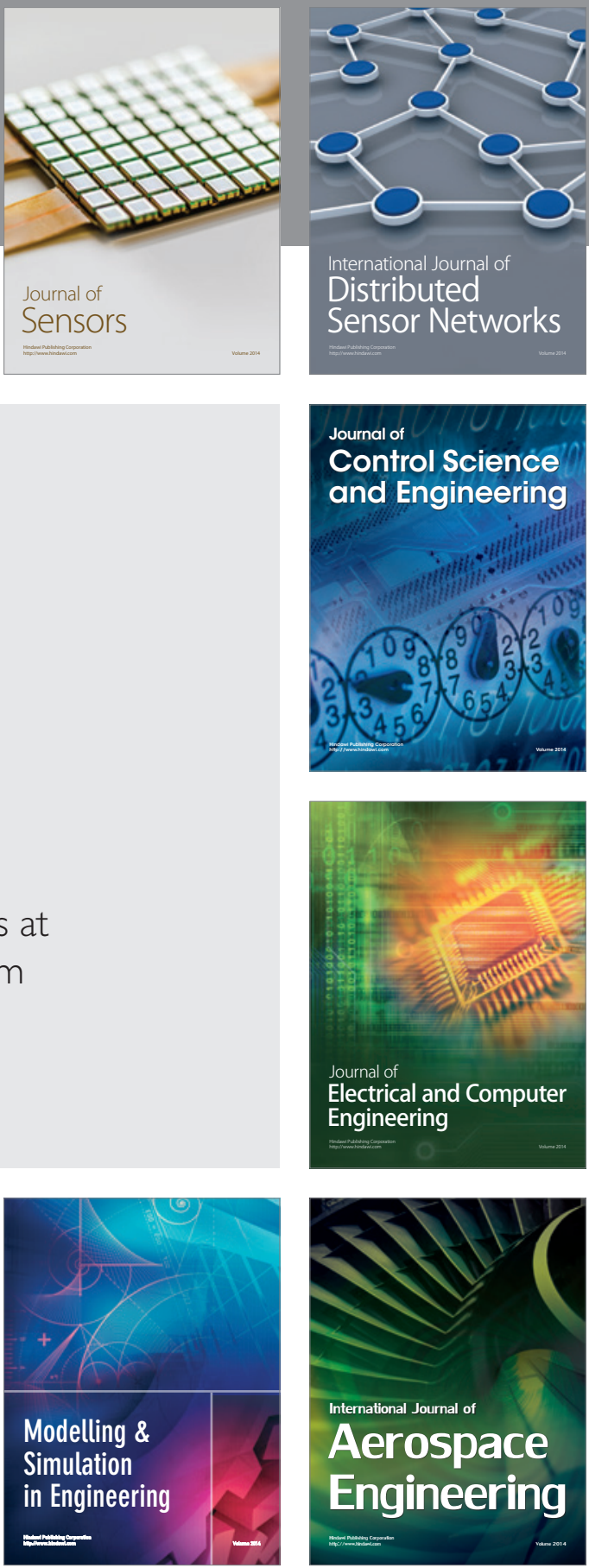

Journal of

Control Science

and Engineering
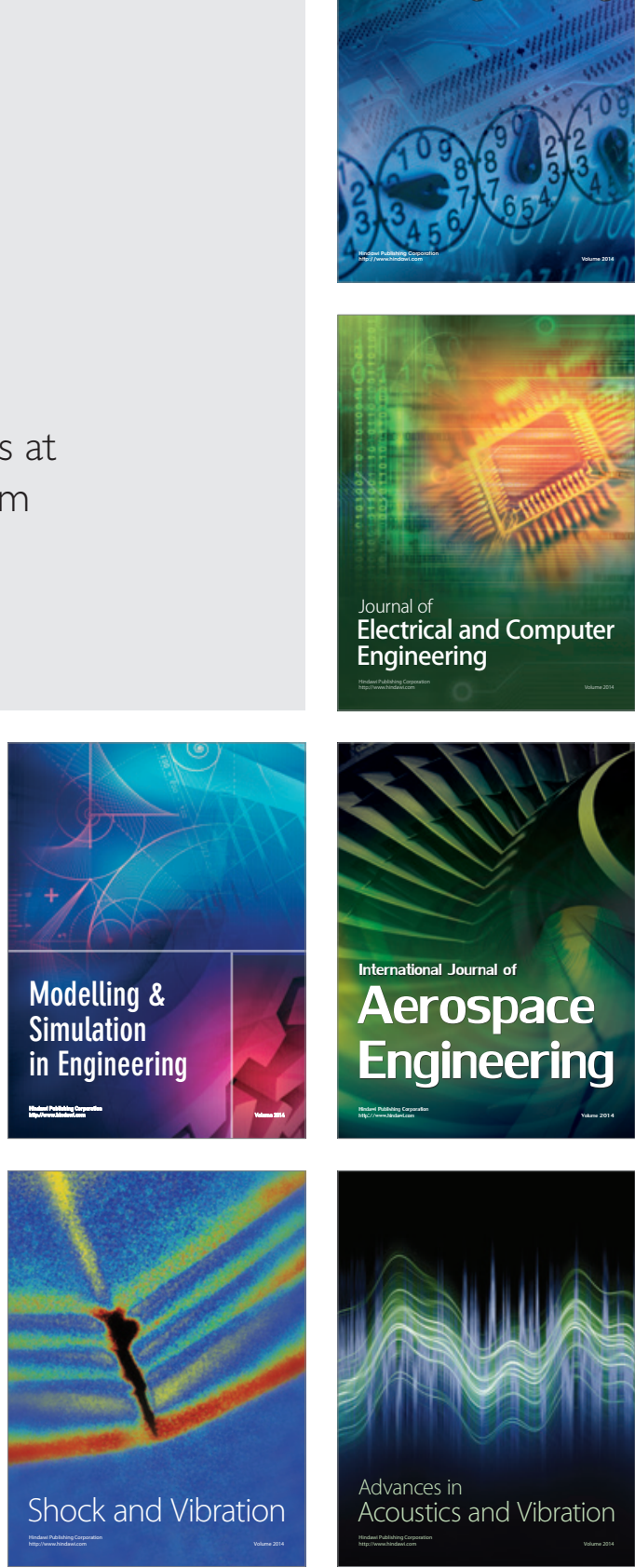